\title{
Dissociating the phononic, magnetic and electronic contributions to thermal conductivity: a computational study in alpha-iron
}

\author{
S. Nikolov ${ }^{1, *}$ (D), J. Tranchida ${ }^{2}$, K. Ramakrishna ${ }^{3,4}$, M. Lokamani $^{4}$, A. Cangi ${ }^{3,4}$, and \\ M. A. Wood ${ }^{1}$ \\ ${ }^{1}$ Computational Multiscale Department, Sandia National Laboratories, P.O. Box 5800, MS 1322, Albuquerque, NM 87185, USA \\ ${ }^{2}$ CEA, DES/IRESNE/DEC, 13018 Saint Paul Lès Durance, France \\ ${ }^{3}$ Center for Advanced Systems Understanding (CASUS), D-02826 Görlitz, Germany \\ ${ }^{4}$ Helmholtz-Zentrum Dresden-Rossendorf (HZDR), D-01328 Dresden, Germany
}

Received: 30 September 2021

Accepted: 30 December 2021

Published online:

3 February 2022

(C) The Author(s) 2022

\begin{abstract}
Computational tools to study thermodynamic properties of magnetic materials have, until recently, been limited to phenomenological modeling or to small domain sizes limiting our mechanistic understanding of thermal transport in ferromagnets. Herein, we study the interplay of phonon and magnetic spin contributions to the thermal conductivity in $\alpha$-iron utilizing non-equilibrium molecular dynamics simulations. It was observed that the magnetic spin contribution to the total thermal conductivity exceeds lattice transport for temperatures up to two-thirds of the Curie temperature after which only strongly coupled magnon-phonon modes become active heat carriers. Characterizations of the phonon and magnon spectra give a detailed insight into the coupling between these heat carriers, and the temperature sensitivity of these coupled systems. Comparisons to both experiments and ab initio data support our inferred electronic thermal conductivity, supporting the coupled molecular dynamics/spin dynamics framework as a viable method to extend the predictive capability for magnetic material properties.
\end{abstract}

\section{Introduction}

Spin-lattice coupling effects in micromagnetic materials are critically important for a myriad of material applications, including the development of nano- sensors [1-4], spintronic logic devices [5-8] and high entropy alloys [9-12]. The ultrafast magnetic switching in Gd-Fe ferrimagnets, for example, arises from a mismatch in the magnetic sublattices, which are coupled by an antiferromagnetic exchange interaction. Such ultrafast switching can be leveraged to

Handling Editor: M. Grant Norton.

Address correspondence to E-mail: snikolo@sandia.gov 
create spintronic devices with exceptionally high recording densities $\left(\mathrm{Tb} / \mathrm{inch}^{2}\right.$ ) and writing rates (fsps scale), paving the way for future advancements in quantum and neuromorphic computing [13-15].

Spin-lattice coupling effects can also be leveraged for dynamic heat flow control in ferromagnetic insulators, enabling the creation of novel nanoscale thermal management strategies [16, 17]. Unlike typical insulators where thermal conductivity can be largely inferred solely from the phonons, in ferromagnetic insulators the heat carriers involved are the phonons, electrons, and magnons. Given that magnons are highly sensitive to the magnetic configuration as well as any external magnetic fields, changes in these properties can be exploited to vary the underlying thermal conductivity and regulate heat flow within the material. Spin caloritronic efforts like this can make both future and existing electronic devices more robust, due to a fine control of the temperature gradients within the material [18]. Unfortunately, the ability to directly gauge the coupling between spin and heat currents is limited by a lack of available computational tools.

In the past, methods based on density functional theory (DFT) $[19,20]$ have been the overwhelmingly popular approach to probe the underlying spin structures in micromagnetic materials [21, 22]. These methods, although highly accurate, do not scale well with increasing system size and temperature. As such many of these $a b$ initio calculations have been performed at zero temperature [23, 24]. Continuum micromagnetic codes can handle much larger temporal and spatial domains but their shortcomings stem from the underlying continuum assumptions which make it difficult to capture complex physics arising from material impurities, material defects, surface anisotropies, and ultrafast magnetization dynamics $[25,26]$.

Alleviating both of the aforementioned shortcomings, atomistic spin-lattice models easily handle complex microstructures and their dynamics while still offering access to spatio-temporal domains that are orders of magnitude larger than what $a b$ initio calculations can handle [25]. In addition to this, atomistic spin-lattice codes naturally couple to MD and DFT codes given each are particle-based computational methods. The ability to simultaneously capture the electronic, phononic and magnetic degrees of freedom within a material remains a significant modelling and simulation challenge.
In the last few years, a number of researchers have focused on incorporating spin-lattice effects into molecular dynamics simulations in this manner [27-31]. These coupled molecular dynamics/spin dynamics (MD-SD) schemes employ the stochastic Landau-Lifshitz-Gilbert equations of motion, which describe how the spin angular velocity evolves in time when the spins are coupled to a thermal bath obeying the fluctuation-dissipation theorem [32, 33]. To achieve spin-lattice coupling, a lattice-dependent exchange interaction is incorporated into the magnetic Hamiltonian which describes the total energy of the magnetic spin subsystem. Parameterization of the exchange interaction is typically based on a BetheSlater function, which is fitted to $a b$ initio data [31, 34, 35]. Similar parameterization procedures are employed to capture the phononic degrees of freedom (interatomic potential) wherein machine learned model forms can be trained to reproduce $a b$ initio level accuracy, albeit at a drastically reduced computational cost [36]. The proper combination of quantum accurate interatomic potentials with a complex spin Hamiltonian (i.e., that avoids double counting) solves a grand challenge in magnetic materials modelling and opens the door for a myriad of research outlets [37].

In the present work, we probe the impact of spinlattice coupling on the magnon and phonon thermal conductivities of $\alpha$-iron in the $300-1200 \mathrm{~K}$ range. Within this effort, we also employ existing experimental data for the total thermal conductivity of iron to infer the corresponding changes in electronic conductivity with temperature. In addition to the thermal conductivity measurements, a complementary spectral analysis of the phonon and magnon heat carrying modes in iron is carried out. We hypothesize that the spin contribution to thermal conduction is non-negligible, even in electrically conductive metals like iron. Thus, the present novel approach for modelling magnetic materials using coupled MD-SD simulations gives us a first of its' kind opportunity to examine the impact that magnetic disorder has on the heat currents in (ferro-)magnetic materials.

\section{Methods}

Large scale MD-SD simulations have been carried out using the SPIN package of the open-source LAMMPS software $[33,38]$ in order to perform true thermo- 
mechanical property predictions of the $\alpha$-phase of iron. The interatomic potential used in the current work is a Spectral Neighborhood Analysis Potential (SNAP), which has been parameterized specifically for MD-SD simulations [37]. As noted in our earlier work, this potential was trained to reproduce the magnetoelastic behavior of $\alpha$-phase iron $(<20 \mathrm{GPa}$ and $<1200 K)$ [37]. Additionally, the spin Hamiltonian was parameterized using a set of non-collinear magnetic DFT calculations, which were carried out in VASP [39-42]. Further details regarding the optimization and training of the SNAP potential and spin Hamiltonian can be found in Nikolov et al. [37]. A brief summary of the MD, SD, and MD-SD schemes is given in appendix A.

We measure the thermal conductivity directly in terms of the heat flux once a steady thermal gradient is established [43, 44]. In order to gauge how the thermal conductivity varies throughout the $\alpha$-phase of iron, we conduct non-equilibrium molecular dynamics (NEMD) [45, 46] using triply periodic $20 \times 20 \times 240$ unit cell replicas $(\sim 5.75 \times 5.75 \times 68.9 \mathrm{~nm}$ with 192,000 atoms), with two $20 \times 20 \times 20$ hot/cold sections $(\sim 5.75 \times 5.75 \times 5.75 \mathrm{~nm})$. The hot $/$ cold sections where thermostats are applied are spaced 100 cells $(\sim 28.8 \mathrm{~nm})$ apart, an illustration of the computational setup is shown in Figure 1. Thermal analysis is conducted under the pressure-controlled conditions (PCC), as outlined in our earlier work [37]. The PCC scheme assumes that the spin and lattice target temperatures in their respective Langevin thermostats are equal: $T_{l}=T_{s}$. Thus for brevity from this point forward we drop the spin/lattice notation in the temperature variable and just assume $T=T_{l}=T_{s}$. To establish a temperature gradient within the bar, the temperature of the hot section is set as $T_{\max }=1.08 T_{\min }$ where herein $T_{\min }$ is varied over the range of $300-1200 \mathrm{~K}$.

In order to maintain the cold/hot sections at the target temperatures $T_{\min } / T_{\max }$, we apply Langevin thermostats to both the atoms and spins [33]. The region between the hot/cold section is not thermostatted and evolves in the microcanonical ensemble (NVE). The heat flux can be calculated by tracking the energies which are added/removed by either thermostat. For a Langevin thermostat, the net energy added is the sum of energy added by the random forces $(\boldsymbol{f}$ and $\boldsymbol{\eta}(t))$ and the energy removed by the dampening forces (whose scale is set by $\gamma_{L}$ and $\lambda$ ), see appendix A for details. Once the heat fluxes are known, Fourier's law allows us to determine the corresponding thermal conductivities. For a given average temperature $T_{\text {ave }}=1.04 T_{\text {min }}$, the lattice constant used to scale the computational box is based on our earlier results for the PCC scheme [37], which takes thermal expansion into account. Since the PCC scheme underpredicts the Curie transition [37], the reported temperatures are rescaled following the procedure outlined by Evans et al. [47].

To equilibrate each system, we run dynamics until the average temperature in the non-thermostated regions stabilizes to $T_{\text {ave }}=1.04 T_{\min }$. All simulation geometries start out initially at $0 \mathrm{~K}$, it was observed the total equilibration time varies from $200-1000$ ps depending on $T_{\min }$, where higher temperatures take longer to equilibrate. Since the material is allowed to thermally expand, in all cases our samples equilibrate to pressures near $0 \mathrm{GPa}\left(p_{\text {eq }}<0.1 \mathrm{GPa}\right)$. Once the temperature gradient in the non-thermostatted section has been equilibrated the simulation is continued for another $1000 \mathrm{ps}$ in order to determine the heat fluxes for both the phonon and magnon sub-systems via their respective thermostats. The dampening constant for the MD thermostat is set to 1 picosecond; meanwhile, the dampening for the SD thermostat is set to 0.01 (dimensionless). The timestep in all simulations is set to 0.0005 ps and the magnitude of the magnetic spin vectors is set to $2.2 \mathrm{Bohr}$ magneton.

The phonon density of states (DOS) and magnon norm/angular velocity DOS spectra calculations are performed using a $20 \times 20 \times 20$ simulation cell which is thermostated (both atomic and spin thermostats are applied) to the average bar temperature, $T_{\text {ave }}=$ $1.04 T_{\min }$ for $150 \mathrm{ps}$. Subsequently, the atomic velocity, spin norms, and precessional velocities are outputted when the thermostats have been removed (NVE dynamics) for every atom/spin at 2.5 fs intervals for $100 \mathrm{ps}$, leading to a frequency space discretization of $0.01 \mathrm{THz}$ when these time series data are fast Fourier transformed (FFT). The FFT is defined as

$$
F F T[X(t)](k)=\sum_{t=1}^{n} X(t) W_{n}^{(t-1)(k-1)},
$$

where $F F T[X(t)](k)$ represents the discrete Fourier transform of a given time series vector $X(t)$. Here, $t$ and $k$ represent the time and frequency domains, respectively, and $W_{n}=e^{(-2 \pi i) / n}$ is one of $n$ roots of unity. As a consistency check, we also tested a frequency resolution of $0.002857 \mathrm{THz}$, for which we 
Figure 1 Illustration of the computational setup for thermal conductivity measurements. The heat flux for both the lattice and spins is calculated between thermal reservoirs, which in conjunction with the temperature gradient defines the conductivity via Fourier's law.

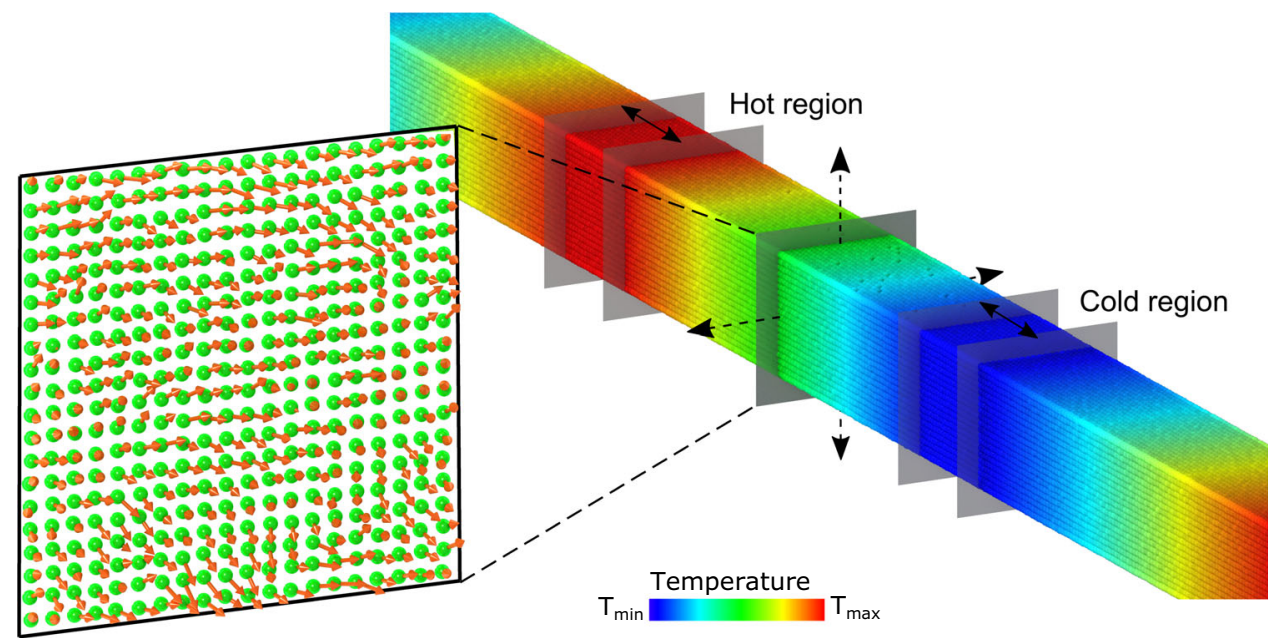

found no discernible change in the phonon DOS and magnon spectra. We calculate the phonon DOS

$$
\Psi(k)=\frac{\psi(k)}{\int \psi(k) d k}
$$

from the discrete Fourier transform of each atom's velocity components in the $\mathrm{x}, \mathrm{y}$, and $\mathrm{z}$ directions.

$$
\begin{aligned}
\psi(k) & =\frac{1}{3 M}\left[\sum_{i=1 \ldots M}\left|F F T\left[v_{x, i}(t)\right]\right|+\left|F F T\left[v_{y, i}(t)\right]\right| \ldots\right. \\
& \left.+\left|F F T\left[v_{z, i}(t)\right]\right|\right]
\end{aligned}
$$

Meanwhile, for the magnons, we look at the frequency domain representation of both the spin orientation $(\vec{s})$ and precessional velocity along the $z$ direction $\left(\omega_{z}\right)$, which corresponds to the direction of initial magnetic alignment in the sample. Figure 2 illustrates graphically how the $\vec{s}$ and $\vec{\omega}$ vectors vary in time. The magnon power spectral density (PSD)

$$
\Phi(k)=\frac{\phi(k)}{\int \phi(k) d k},
$$

is computed from the spin projections

$$
\begin{aligned}
\phi(k)= & \frac{1}{3 M}\left[\sum_{i=1 \ldots M}\left|F F T\left[s_{x, i}(t)\right]\right|+\left|F F T\left[s_{y, i}(t)\right]\right| \ldots\right. \\
& \left.+\left|F F T\left[s_{z, i}(t)\right]\right|\right]
\end{aligned}
$$

Finally, the angular velocity spectrum is defined as
$\Theta(k)=\frac{\theta(k)}{\int \theta(k) d k}$,

where

$$
\theta(k)=\frac{1}{3 M}\left[\sum_{i=1 \ldots M}\left|F F T\left[\omega_{z, i}(t)\right]\right|\right]
$$

Here, $M$ denotes the number of atoms/spins in the sample. Each spectrum is normalized to allow for ease of comparison between different temperatures.

The $a b$ initio predictions of the electronic thermal conductivity are carried out using the Kubo-Greenwood formalism [47-51] based on Kohn-Sham orbitals and eigenvalues obtained from DFT [20] calculations which are performed with VASP [39-42]. The unit cell contains 16 iron atoms with a grid consisting of $4 \times 4 \times 4 k$-points on a total of 160 bands. A PAW pseudopotential [52] consistent with the PBE [53] exchange-correlation (XC) functional with a core radius of $r_{c}=1.9 a_{B}$ and containing 16 valence electrons is used in all the DFT calculations. The plane wave cutoff is set to $750 \mathrm{eV}$ and the convergence in each self-consistency cycle is set to $10^{-5}$. A modified version of the KG4VASP package [54] is used for the post-processing of the electrical conductivity tensor terms. The electronic component of the thermal conductivity is evaluated from the dynamic Onsager coefficients in the DC limit $(\omega \rightarrow 0)$, see Ref. [55] for further details. These calculations were performed on the basis of six random ionic configurations taken from the equilibrated MD configurations which were obtained from DFT-MD calculations performed with VASP [39-42]. 


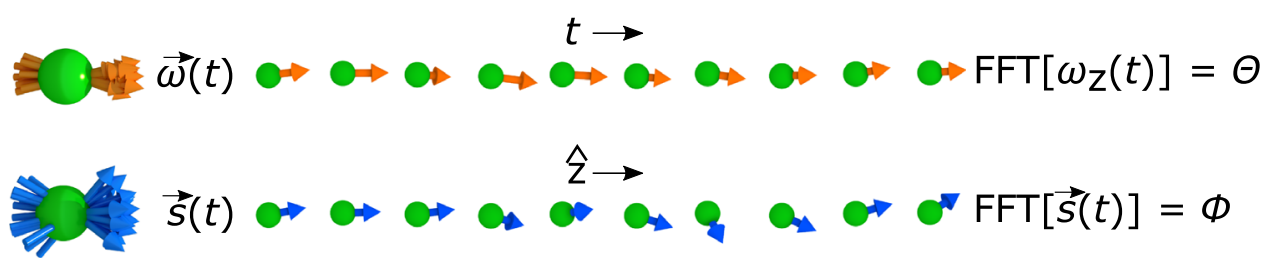

Figure 2 Comparison between $\vec{\omega}$ and $\vec{s}$ at different points in time for $T=380 \mathrm{~K}$. Deviation from the magnetization axis $(\hat{z})$ is much smaller for $\vec{\omega}$.

\section{Results}

Figure 3a) shows the predicted thermal conductivity obtained from MD, SD, and coupled MD-SD simulations. Comparing phonon data between MD (blue dashed) and MD-SD models (blue and red markers), we infer that the inclusion of spin dynamics does alter the phonon thermal conductivity through the coupling between phonons and magnons, leading to a slight decrease in the phonon thermal conductivity. In the SD case (where atoms do not vibrate), the magnon thermal conductivity (red dashed) at low temperatures (near $380 \mathrm{~K}$ ) is approximately three times larger than the MD-SD magnon conductivity (red markers). As the temperature increases, the SD magnon thermal conductivity rapidly decreases, approaching values much closer to the MD-SD magnon conductivities. Based on this we can infer that the impact of lattice motion tends to be more significant at temperatures below $700 \mathrm{~K}$ where only mild spin fluctuations are present $(m>0.88)$, leaving magnon-phonon scattering to explain the sharp decrease in conductivity. At temperatures above $700 \mathrm{~K}$ spin fluctuations become significantly higher as the net magnetization in the simulation cell approaches zero and the significance of lattice vibrations becomes weaker. For the MD-SD case, the magnon thermal conductivity is larger than the phonon thermal conductivity in the range of $<700 \mathrm{~K}$. At higher temperatures, the magnon conductivity decreases below that of the phonons and near the Curie temperature it approaches values close to zero $\left(\kappa_{m, M D-S D} \approx 1-2 \mathrm{~W} / \mathrm{mK}\right)$.

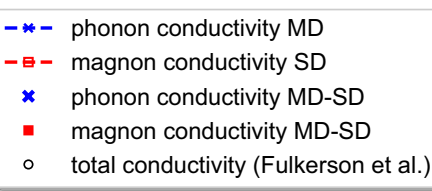

(a)

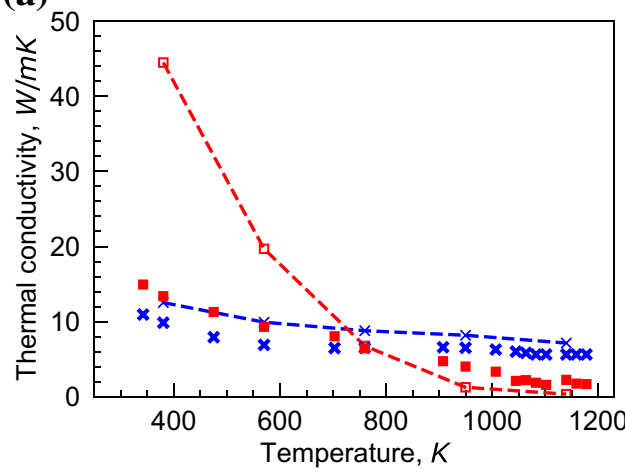

Figure 3 a Comparison of thermal conductivity data between spin-dynamics only (SD), lattice-dynamics only (MD), and coupled molecular dynamics/spin dynamics (MD-SD) simulations. SD simulations show a markedly larger change in thermal conductivity between $400-1200 \mathrm{~K}$ compared to the MD-SD results indicating the lattice motion has a strong impact magnon scattering. Conversely, phonon conductivity decreases only
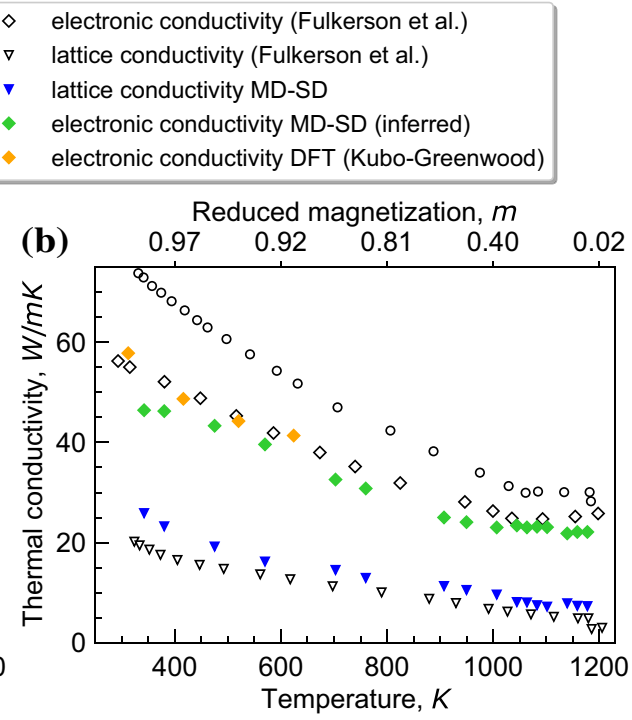

slightly with the inclusion of spin dynamics. $\mathbf{b}$ MD-SD thermal conductivity and corresponding experimental data from Fulkerson et. al. [56]. Kubo-Greenwood DFT calculations, performed following the MD-SD predicted thermal expansion, validate the $\mathrm{SNAP}+$ Spin model construction and agree well with available experiments. 
Fig. $3 b)$ shows the MD-SD thermal conductivity results along with a complementary experimental data set (black markers) from Fulkerson et al. [56]. In order to better compare MD-SD results with the data from experiment, we assume that the total thermal conductivity measured in experiment can be expressed in terms of an electronic and lattice thermal conductivity as $\kappa_{T}=\kappa_{l}+\kappa_{e}$, where the lattice thermal conductivity is composed of the phonon $\kappa_{p}$ and the magnon $\kappa_{m}$ thermal conductivities, $\kappa_{l}=\kappa_{p}+\kappa_{m}$. By using the experimental values for the total thermal conductivity $\kappa_{T, \text { exp }}$, we can use the phonon and magnon MD-SD thermal conductivities to infer an electronic thermal conductivity for our MD-SD simulations as: $\kappa_{e, M D-S D}=\kappa_{T, e x p}-\kappa_{p, M D-S D}-\kappa_{m, M D-S D}$.

The inferred $\kappa_{e, M D-S D}$ (green markers) is compared against the experimental data in Fig. 3b). Our inferred electronic thermal conductivity is in good agreement with the experimental data which was obtained using electrical resistivity measurements. Moreover, the inferred lattice thermal conductivity which Fulkerson calculates using the Backlund et al. relation [57] is in good agreement with the MD-SD lattice conductivity data. Fulkerson et al. experimentally shows that past the Curie temperature the electronic thermal conductivity data experiences a change in slope, as it begins to flatten out. This trend is also observed in our MD-SD simulations due to the loss of magnon thermal conductivity. As Buckland points out the inverse of the electronic thermal conductivity gives a measure of the electronic thermal resistivity, which can be separated into terms due to thermal scattering $\left(W_{e T}\right)$, s-d scattering $\left(W_{e S}\right)$, and impurity scattering $\left(W_{e I}\right)$ [57]. Since at low temperatures the thermal scattering term dominates [57], we estimate that $W_{e T} \approx 2 \mathrm{~cm}-\mathrm{K} / \mathrm{W}$, which is $33 \%$ higher than the value found by Backlund [57].

Note that the current SNAP potential is not trained to reproduce the bcc-fcc transition, so the discontinuity observed experimentally in the lattice thermal conductivity near $1180 \mathrm{~K}$ cannot be reproduced with the current interatomic potential. However, this can be easily fixed in the future by including additional DFT training data [37].

The orange markers in Fig. 3b) represent the electronic contribution to the thermal conductivity obtained from $a b$ initio calculations using the KuboGreenwood formalism. These calculations have been successfully used to evaluate the behavior of iron [58] and its alloys especially for the transport properties under earth-core conditions [59, 60]. The KuboGreenwood evaluations are performed at the MD-SD predicted lattice constants. Fig. 3b) shows the DFT calculations are in great agreement with experimental data. Moreover, above $400 \mathrm{~K}$, the DFT calculations are also in reasonable agreement with the MD-SD data. Interestingly, below $400 \mathrm{~K}$, the MD-SD data overestimate the lattice conductivity $\left(\kappa_{p, M D-S D}+\kappa_{m, M D-S D}\right)$ which in turn leads to a decrease in $\kappa_{e, M D-S D}$.

To understand the temperature dependence and potential scattering mechanisms of either heat carrier type in our MD-SD simulations, we turn to a spectral analysis of the heat carrying modes in iron. The plot in Fig. 4a) shows phonon DOS data for three different temperatures (solid blue, green, and red) along with an experimental data set (black markers) gathered using a nuclear resonant inelastic X-ray scattering (NRIXS) technique at ambient conditions (values scaled for clarity) [61]. The experimentally predicted acoustic and optical peaks are near approx. 5.6 and $8.5 \mathrm{THz}$, respectively, and are matched reasonably well by the MD-SD data. The MD-SD simulations tend to overestimate the acoustic band edge peak by about $0.4 \mathrm{THz}$ or $1.65 \mathrm{meV}$ and do not show the small optical peak near $6.6 \mathrm{THz}$, observed by Minkiewicz [62] and $\mathrm{Hu}$ [63]. Increasing the frequency resolutions from 0.01 to $0.002857 \mathrm{THz}$ did not lead to any discernible changes in the DOS curves. For the MD-SD results, as the temperature increases the DOS broadens as the acoustic peaks shift to lower energies with thermal expansion, consistent with previous findings [64-66]. The inset in Figure 4a) shows how the first peak (acoustic band edge) shifts at higher temperatures. Overall the MD-SD simulations do a good job of estimating the frequency of the acoustic peak within the range of $300-1000 \mathrm{~K}$ indicating the PCC scheme captures thermal expansion at higher temperatures.

Figure $4 \mathrm{~b}$ ) shows how the MD-SD magnon PSD in the frequency domain varies at different temperatures. As the temperature increases a uniform red shift and broadening of the magnon modes is observed, consistent with prior studies [67]. As the Curie temperature is approached, and the magnon thermal conductivity decreases, many higher frequency peaks diminish, owing to a loss of short ranged spin ordering. The inset of Figure $4 \mathrm{~b}$ ) shows the high frequency decay of the magnon PSD. It can 


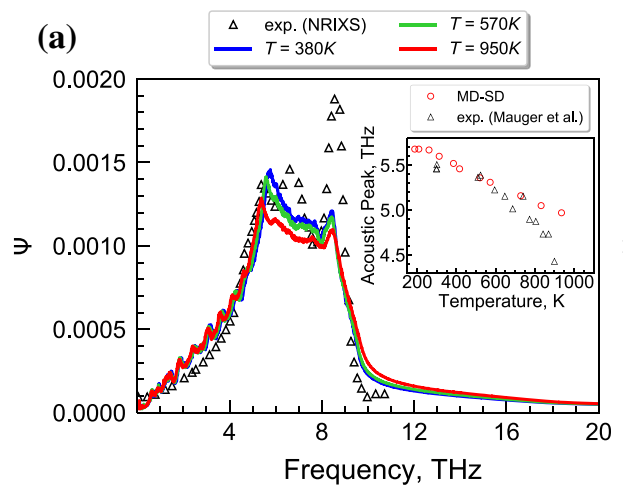

Figure 4 a Phonon density of states for MD-SD simulations along with experiments at ambient conditions obtained via nuclear resonant inelastic X-ray scattering [61]. At $T=312 K$, the location of the acoustic and optical peaks matches the experimental measurements well while increasing temperatures cause noticeable

be seen that the magnons remain only weakly identifiable at higher temperatures compared to the phonons [29] and only at low temperatures is a peak observed near 46-47 THz.

To better compare between the MD-SD, MD, and SD cases, we also examine how the phonon DOS and magnon spectra $(\Phi)$ compare at a temperature of $T=570 \mathrm{~K}(m=0.786)$, shown in Fig. 5. Fig. 5a) shows that while the phonon acoustic peaks remain at the same frequency, a small redshift of about $0.24 \mathrm{THz}$ in the optical band edge peak is observed. The inclusion of spin dynamics also seems to dampen out excitations throughout the $0-9 \mathrm{THz}$ range. Conversely, comparing the magnon spectra, $\Phi$, between the MDSD and SD data, no shifts at low frequencies $(<15 \mathrm{THz})$ are observed, as exemplified in Fig. 5b). However, at high frequencies, the addition of lattice motion into the dynamics seems to strongly dampen the high frequency peak near $41 \mathrm{THz}$.

After examining the spin orientation $(\vec{s})$ spectra $\Phi$, we turn to the spectra defined by the precession velocities of individual spins $(\vec{\omega})$. Fig. 6 shows how the PSD of the precessional velocity in the $z$-direction (thermal gradient) $\left(\omega_{z}\right)$ varies between MD-SD (solid green) and SD (solid orange) simulations for $m=0.928$. As can be seen for the isolated spins (SD), where the lattice is fixed, $\Theta$ reverts to a profile similar to $\Phi$. Meanwhile, for the coupled MD-SD case, the phonon DOS is noticeably super-imposed in the $\omega_{z}$ signal. While this is noticeable along the longitudinal direction $(z)$, it was found that the transerve directions ( $x$ and $y$ components) of the precessional

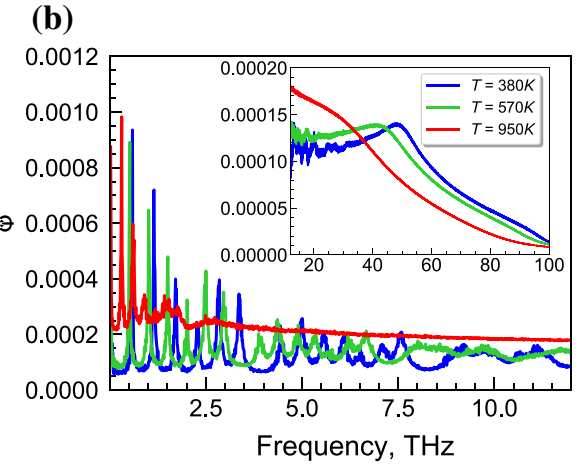

red shifting. $\mathbf{b}$ Normalized magnon spectra in the low frequency range for different temperatures. Significant attenuation of the peaks is observed near the Curie temperature $(T=1045 \mathrm{~K})$, inset shows magnon behavior at high frequencies.

velocity, $\vec{\omega}$, do not show this effect. As Figure 2 shows, the $\vec{s}$ vectors vary rapidly in time, showing a weak coherence with neighboring vectors, whereas the $\vec{\omega}$ vectors are more tightly bound to the $z$-axis and exhibit much stronger coherence with their neighbors.

What is particularly interesting in Fig. 6 are the pronounced magnon peaks below $5 \mathrm{THz}$, which persist even when the lattice is thermalized (MD-SD). A non-magnetic simulation of iron would have only yielded a smooth increase in the phonon DOS up to the acoustic band edge, but this is now decorated with vibrations that show strong overlap in the spin procession and phonon spectra. In fact, many of the distinct peaks in the MD-SD vibrational spectra (except the optical band edge) contain a corresponding peak in the magnon spectra, highlighting strong magnon-phonon coupling in this ferromagnetic material.

The inset of Fig. 6 shows how the $\omega_{z}$ PSD varies with temperature. Interestingly, the strong magnonphonon coupled modes are not picked up by $\omega_{z}$ signal near the Curie temperature. The attenuation of the phonon DOS in $\Theta$ is indicative of the weakening spin-lattice coupling (relative to thermal fluctuations) at higher temperatures. This effect can be inferred also from Callen's law which describes the decay of the anisotropy energies (which largely arise from spin-orbit coupling) at higher temperatures [68]. At higher temperatures, we also observe the same red shift and peak broadening detected in $\Phi$. No discernible peaks for $\Theta$ are found at frequencies above 


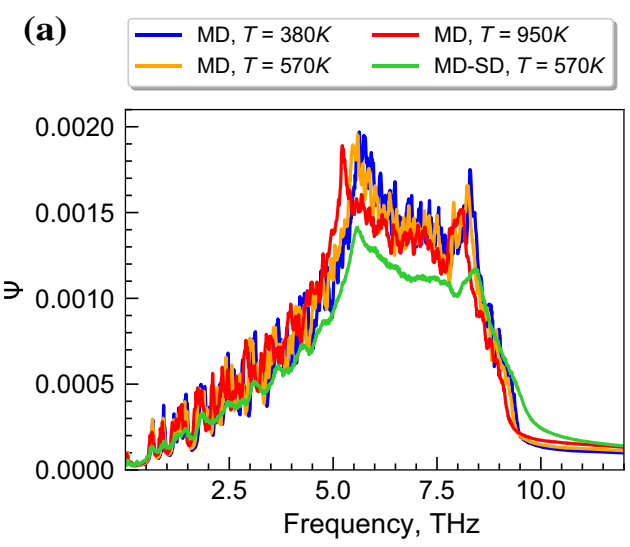

Figure 5 Comparison between MD and MD-SD simulations for phonon $(\Psi)$ and magnon spectra $(\Phi)$. a) At $T=570 \mathrm{~K}$, for both $\mathrm{MD}$ and MD-SD the acoustic band edge peak occurs at $5.6 \mathrm{THz}$. Meanwhile, for the MD-SD data, the optical peak is shifted by $0.23 \mathrm{THz}$ from the corresponding frozen spin, MD peak (at 8.26 $\mathrm{THz}$ ). b) For $T=570 \mathrm{~K}$, the magnon spectra in the low frequency

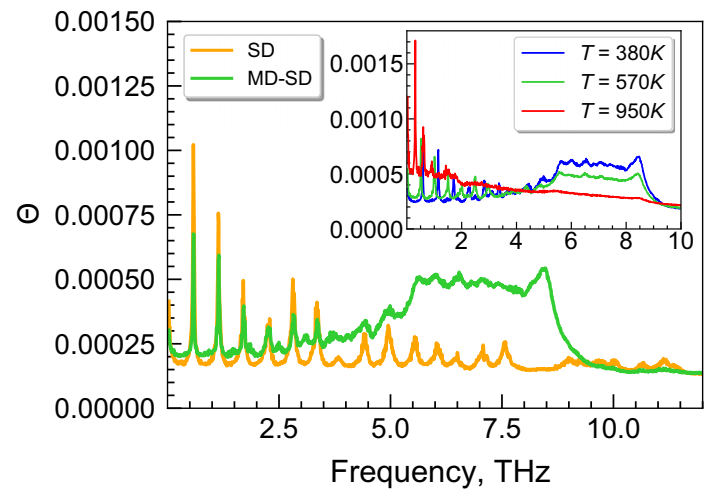

Figure 6 Comparison between MD-SD and SD angular velocity spectra for $T=570 \mathrm{~K}$. In the SD simulations, there are no lattice vibrations so the phonon DOS is not reflected in the angular velocity spectra $\Theta$. Inset plot shows MD-SD data for $\Theta$ at different temperatures. At low temperatures, the phonon DOS peak is reflected in the angular velocity spectra, indicating that the precessional motions of the spins are reflective of phonon vibrations. Upon approaching the paramagnetic state the phonon DOS peaks (in the 5 to $9 \mathrm{THz}$ range) vanish.

$20 \mathrm{THz}$. This contrasts the data for $\Phi$ where at low temperatures a high frequency peak near $41-43 \mathrm{THz}$ can be observed.

At $\mathrm{T}=950 \mathrm{~K}(\mathrm{~m}=0.40)$, while still below the Curie temperature, the superposition of phonon vibrations in the magnon spectra is lost, indicating that at this elevated temperature the two sub-systems of heat carriers are decoupled. However, this separation of the lattice and magnetic degrees of freedom is not absolute, Fig. 3a) shows how the SD magnon

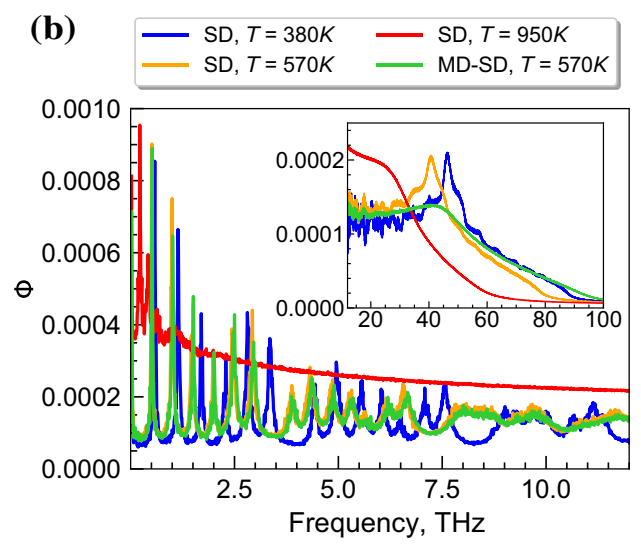

range (0-12 THz) does not change between the SD and MD-SD simulation conditions. However, as shown in the inset plot, near 40 $\mathrm{THz}$ the MD-SD magnon spectra does experience a strong attenuation, indicating that these high frequency vibrations are sensitive to lattice motion.

conductivity is actually below the MD-SD conductivity near $950 \mathrm{~K}$ indicating phonon driven thermal transport in the magnon sub-system. Furthermore, in all temperatures studied here, the phonon conductivity is lower in the coupled MD-SD case relative to the simulation conditions where spins are frozen. In effect, while the additional scattering between the two subsystems decreases the overall phonon conductivity, the strong magnon-phonon coupling gives rise to additional heat carrying spin wave modes that are mediated by persistent phonon modes at high temperatures. Furthermore, it is observed that coherency in the spin state $(m>0.8)$ plays a drastic role in the ability for a material to conduct heat through spin waves, which is evidenced by the rapidly decaying features in the magnon spectra in Figs. 4, 5, 6 .

\section{Conclusion}

The impact of phonon-magnon coupling on the thermal conductivity of iron was quantified up-to and beyond the Curie temperature in ferromagnetic iron. It was found that for the phonon contribution, the inclusion of spin dynamics leads to a small decrease in the thermal conductivity throughout the entire temperature range. Meanwhile, the inclusion of lattice vibrations significantly decreases the magnon thermal conductivity up to $700 \mathrm{~K}$, but remains non-negligible up to the Curie temperature. Both of 
these observed effects are due to the added scattering mechanisms that each of the two sub-systems provides to the other. However, it was observed that the spin contribution to thermal conductivity near the Curie temperature remains non-zero strictly due to spin wave coupling to lattice vibrations.

Utilizing the calculated lattice and spin conductivities along with experimental measurements of the total thermal conductivity, an inferred electronic conductivity of iron is obtained in good agreement with both experimental findings and $a b$ initio calculations. While the classical MD simulations are unable to capture these electronic effects, it is encouraging that our $a b$ initio trained parameterization reproduces a proper partitioning of the thermal conductivity among the phonon, magnon and inferred electronic degrees of freedom.

To supplement the observed trends in thermal conductivity, the phonon and magnon spectra were analyzed to obtain a deeper understanding of how temperature impacts each heat carrying mode. Our findings show that for the phonon DOS, our predictions are in good agreement with nuclear resonant inelastic X-ray scattering data, including peak red shifting with increasing temperature. As expected, approaching the magnetic order-disorder transition, peaks in the magnon spectra become largely featureless. From these spectral results, we were able to highlight changes in the spectra that are due to strong magnon-phonon coupling which helps interpret changes in the coupled MD-SD conductivity relative to the decoupled predictions. A shift in the phonon optical band edge due to spin contributions and the loss of the high-frequency magnon mode where lattice vibrations are present, exemplify these spectral changes.

The efforts described here outline a basis for characterizing the impact spin currents have on the heat flux in any magnetic material. These atomistic simulation methods and analyses can be easily applied to more complicated systems featuring grain boundaries, multiple magnetic domains, defects, and additional alloying elements. Thus, a more mechanistic and holistic view of thermal conductivity in this class of metals can be obtained within one set of simulation tools. Our future work is aimed at utilizing these methods to explore these complexities as well as phase transitions in magnetic materials.

\section{Acknowledgements}

Sandia National Laboratories is a multimission laboratory managed and operated by National Technology and Engineering Solutions of Sandia, LLC., a wholly owned subsidiary of Honeywell International, Inc., for the U.S. Department of Energy's National Nuclear Security Administration under contract DENA-0003525. This paper describes objective technical results and analysis. Any subjective views or opinions that might be expressed in the paper do not necessarily represent the views of the U.S. Department of Energy or the United States Government. K.R. and A.C. acknowledge funding by the Center for Advanced Systems Understanding (CASUS) which is financed by the German Federal Ministry of Education and Research (BMBF) and by the Saxon Ministry for Science, Culture and Tourism (SMWK) with tax funds on the basis of the budget approved by the Saxon State Parliament. M.L. was supported by the German Federal Ministry of Education and Research (BMBF, No. 01/S18026A-F) by funding the competence center for Big Data and AI "ScaDS.AI Dresden/ Leipzig."

\section{Code Availability}

The code which was used to train the SNAP potential used herein and reported in Ref. [68] is available from https://github.com/FitSNAP/FitSNAP.

\section{Declarations}

Conflict Of Interest Statement The authors declare that they have no known competing financial interests that could have appeared to influence the work reported in this paper.

Open Access This article is licensed under a Creative Commons Attribution 4.0 International License, which permits use, sharing, adaptation, distribution and reproduction in any medium or format, as long as you give appropriate credit to the original author(s) and the source, provide a link to the Creative Commons licence, and indicate if changes were made. The images or other third party material in this article are included in the article's Creative Commons licence, unless indicated otherwise in a credit line to the material. If material is not included in the article's Creative Commons licence and your intended use is 
not permitted by statutory regulation or exceeds the permitted use, you will need to obtain permission directly from the copyright holder. To view a copy of this licence, visit http://creativecommons.org/licen ses/by $/ 4.0 /$.

\section{Appendix}

\section{A Spin-Lattice dynamics}

In the current work for the SD and MD-SD schemes, we utilize a biquadratic spin Hamiltonian which has previously been shown to be a good descriptor for spin behavior in ferromagnets $[69,70]$. The form of the biquadratic spin Hamiltonian is shown in equation (8).

$$
\begin{aligned}
\mathcal{H}_{S D}= & -\sum_{i \neq j}^{N} J\left(r_{i j}\right)\left[\boldsymbol{s}_{i} \cdot \boldsymbol{s}_{j}-1\right] \ldots \\
& -\sum_{i \neq j}^{N} K\left(r_{i j}\right)\left[\left(\boldsymbol{s}_{i} \cdot \boldsymbol{s}_{j}\right)^{2}-1\right]
\end{aligned}
$$

Here, $r_{i j}$ is the interatomic distance between atoms $i$ and $j$. The variables $s_{i}$ and $s_{j}$ are unit vectors representing the orientation of classical atomic spins located on atoms $i$ and $j$. The magnetic exchange functions $J\left(r_{i j}\right)$ and $K\left(r_{i j}\right)$ are defined based on an approximation of the Bethe-Slater curve [71, 72], shown in equation (9).

$f(r)=4 \alpha\left(\frac{r}{\delta}\right)^{2}\left(1-\gamma\left(\frac{r}{\delta}\right)^{2}\right) e^{-\left(\frac{r}{\delta}\right)^{2}} \Upsilon\left(R_{c}-r\right)$

Here, the variables $\alpha$ and $\delta$ are the interaction energy and interaction decay length and $\gamma$ is a dimensionless curvature parameter. Meanwhile, $\Upsilon\left(R_{c}-r\right)$ is Heaviside step function which switches at the radial cutoff

Table 1 Parameters of the biquadratic exchange Hamiltonian for $\mathrm{BCC}$ iron

\begin{tabular}{ll}
\hline MD-SD model parameters & \multicolumn{1}{c}{$\mathrm{BCC} \mathrm{Fe}$} \\
\hline$R_{\text {cut }}$ & $5.0 \AA$ \\
$\alpha_{J}$ & $0.2827 \mathrm{eV}$ \\
$\gamma_{J}$ & -4.747 \\
$\delta_{J}$ & $0.7810 \AA$ \\
$\alpha_{K}$ & $-0.03619 \mathrm{eV}$ \\
$\gamma_{K}$ & -2.973 \\
$\delta_{K}$ & $0.5273 \AA$ \\
\hline
\end{tabular}

$R_{c}$. The corresponding values of $\alpha, \delta$, and $\gamma$ for $J\left(r_{i j}\right)$ and $K\left(r_{i j}\right)$ along with the value of $R_{c}$ for the exchange interaction are shown in Table 1.

As shown in equation (10), the MD-SD Hamiltonian incorporates $\mathcal{H}_{S D}$ from equation (8). The second term on the right side of the equation represents the kinetic energy and the third term $\left(V_{S N A P}\left(r_{i j}\right)\right)$ arises from the interatomic SNAP potential. The variables $\boldsymbol{p}$ and $m_{i}$ are the particle momentum and mass of atom $i$, respectively.

$$
\begin{aligned}
\mathcal{H}_{M D-S D}(\boldsymbol{r}, \boldsymbol{p}, \boldsymbol{s})= & \mathcal{H}_{S D}(\boldsymbol{r}, \boldsymbol{s})+\sum_{i=1}^{N} \frac{|\boldsymbol{p}|^{2}}{2 m_{i}} \ldots \\
& +\sum_{i, j=1}^{N} V_{S N A P}\left(r_{i j}\right)
\end{aligned}
$$

Using the generalized spin-lattice Poisson bracket as defined by Yang et al. [73], along with equation (10), the equations of motion shown in (11)-(13) can be derived.

$\frac{d \boldsymbol{r}_{i}}{d t}=\frac{\boldsymbol{p}_{i}}{m_{i}}$

$$
\begin{aligned}
\frac{d \boldsymbol{p}_{i}}{d t}= & \sum_{j, i \neq j}^{N}\left[-\frac{d V_{S N A P}\left(r_{i j}\right)}{d r_{i j}}+\frac{d J\left(r_{i j}\right)}{d r_{i j}}\left(\boldsymbol{s}_{i} \cdot \boldsymbol{s}_{j}\right)+\ldots\right. \\
& \left.\frac{d K\left(r_{i j}\right)}{d r_{i j}}\left(\boldsymbol{s}_{i} \cdot \boldsymbol{s}_{j}\right)^{2}\right] \boldsymbol{e}_{i j}-\frac{\gamma_{L}}{m_{i}} \boldsymbol{p}_{i}+\boldsymbol{f}(t) \\
\frac{d \boldsymbol{s}_{i}}{d t}= & \frac{1}{\left(1+\lambda^{2}\right)}\left[\left(\boldsymbol{\omega}_{i}+\boldsymbol{\eta}(t)\right) \times \boldsymbol{s}_{i}+\ldots\right. \\
& \left.\lambda \boldsymbol{s}_{i} \times\left(\boldsymbol{\omega}_{i} \times \boldsymbol{s}_{i}\right)\right]
\end{aligned}
$$

Equations (11)-(12) describe how the derivatives of position and momentum change with time. Equation (13) is the Landau-Lifshitz-Gilbert equation which describes spin vector precession in a spin subsystem coupled to a thermal bath. The variable $\omega_{i}$ is the precessional angular velocity whose definition is based on the work of Evans et al. [74]. The definition for $\omega_{i}$ is given in equation (14) below, where $\mu_{i}$ is the atomic spin norm (set to $2.2 \mathrm{Bohr}$ magneton) and $\beta$ is the gyromagnetic ratio $\left(\beta \approx 0.176 \mathrm{rad} \cdot \mathrm{THz} \cdot \mathrm{T}^{-1}\right)$.

$$
\boldsymbol{\omega}_{i}=\frac{\beta}{\mu_{i}} \sum_{j}^{N_{i}} J\left(r_{i j}\right) \boldsymbol{s}_{j}+K\left(r_{i j}\right)\left(\boldsymbol{s}_{i} \cdot \boldsymbol{s}_{j}\right) \boldsymbol{s}_{j}
$$

The variable $\gamma_{L}$ in equation (12) is the Langevin damping constant for the lattice which is coupled to the fluctuating force $f$ (which follows Gaussian 
Table 2 Computational performance of different simulation methods

\begin{tabular}{|c|c|c|c|c|c|c|c|}
\hline Method & Workload balance & $\begin{array}{l}\text { Performance } \\
\text { / s) }\end{array}$ & (atoms $\cdot$ ts & $\begin{array}{l}\text { Number of } \\
(N)\end{array}$ & atoms & $\begin{array}{l}\text { Approx. } \\
\text { scaling }\end{array}$ & Hardware \\
\hline $\begin{array}{l}\text { MD- } \\
\text { SD }\end{array}$ & $\begin{array}{l}266.7 \text { atoms / cpu- } \\
\text { core }\end{array}$ & $2,615,270$ & & 192,000 & & $N^{1}$ & $\begin{array}{l}40 \text { x E5-2695 v4@ } \\
2.10 \mathrm{GHz}\end{array}$ \\
\hline MD & $\begin{array}{l}266.7 \text { atoms / cpu- } \\
\text { core }\end{array}$ & $4,359,437$ & & 192,000 & & $N^{1}$ & $\begin{array}{l}40 \text { x E5-2695 v4@ } \\
2.10 \mathrm{GHz}\end{array}$ \\
\hline SD & $\begin{array}{l}266.7 \text { atoms / cpu- } \\
\text { core }\end{array}$ & $2,664,806$ & & 192,000 & & $N^{1}$ & $\begin{array}{l}40 \text { x E5-2695 v4@ } \\
2.10 \mathrm{GHz}\end{array}$ \\
\hline $\begin{array}{c}\text { DFT- } \\
\text { MD }\end{array}$ & 4 atoms / gpu & 0.454 & & 16 & & $N^{3}$ & $4 \times$ Tesla V100 (32 GB) \\
\hline
\end{tabular}

statistics) by the fluctuation dissipation theorem as shown in equations (15)-(16) [33].

$$
\begin{aligned}
& \langle\boldsymbol{f}(t)\rangle=0 \\
& \left\langle f_{\alpha}(t) f_{\beta}\left(t^{\prime}\right)\right\rangle=2 k_{B} T_{l} \gamma_{L} \delta_{\alpha \beta} \delta\left(t-t^{\prime}\right)
\end{aligned}
$$

The variables $k_{B}$ and $T_{l}$ are the Boltzmann constant and target lattice temperature, respectively [33]. Similarly, in equation (13), we have the variable $\boldsymbol{\eta}(t)$ which is another fluctuating force (this time for the spin subsystem) that follows Gaussian statistics. Again here $\boldsymbol{\eta}(t)$ is coupled to the transverse dampening constant $\lambda$ via the fluctuation dissipation relation, shown in equations (17)-(18) [33].

$$
\begin{aligned}
& \langle\boldsymbol{\eta}(t)\rangle=0 \\
& \left\langle\eta_{\alpha}(t) \eta_{\beta}\left(t^{\prime}\right)\right\rangle=\frac{2 \pi \lambda k_{B} T_{s} \delta_{\alpha \beta} \delta\left(t-t^{\prime}\right)}{\hbar}
\end{aligned}
$$

Here, $T_{s}$ is the target spin temperature and $\hbar$ is Plank's constant.

\section{B Computational performance}

Simulations for the MD-SD, MD, and SD schemes were carried out using 20 dual-socket E5-2695 v4 @ 2.10GHz nodes (36 cores per nodes). The DFT-MD simulations, meanwhile, were carried out using 4 Tesla V100 GPUs. The performance metrics for our simulations are shown in Table 2. Due to the strong linear scaling of ML-IAP potentials like SNAP, we can greatly accelerate computational performance by using a large number of nodes/processors, thus keeping the atom/cpu-core count low [36]. This degree of parallelization is not typical for potentials like EAM or Lennard-Jones where a breakdown of
30,000 atoms /cpu-core is preferred. The atom/cpucore count in MD-SD and SD simulations is limited (to approx. 200-250 atoms/cpu-core) by the sectoring algorithm which is used within the Suzuki-Trotter decomposition of the spin equations of motion. Table 2 also shows that for the MD-SD and SD methods, the inclusion of spin dynamics leads to an approx. $40 \%$ drop in computational performance. This drop in performance is expected and can be attributed largely to the Suzuki-Trotter decomposition which requires quarter timestep updates. Meanwhile, the standard integration scheme in LAMMPS relies on the Velocity-Verlet algorithm which uses a half timestep updates. Even though the lattice is frozen in the SD scheme, the mechanical contribution from the SNAP potential is still computed in order to obtain the correct pressures/energies. For the DFT-MD method the computational performance scales as $N^{3}$, whereas for the classical schemes a scaling of $N^{1}$ is observed, consistent with previous studies [36, 75].

\section{References}

[1] Chang CS, Kostylev M, Ivanov E (2013) Metallic spintronic thin film as a hydrogen sensor. Appl Phys Lett 102(14):142405

[2] Liu X, Lam K, Zhu K, Zheng C, Li X, Du Y, Liu C, Pong PW (2019) Overview of spintronic sensors with internet of things for smart living. IEEE Trans Magn 55(11):1-22

[3] Mohsen A, Al-Mahdawi M, Fouda MM, Oogane M, Ando Y, Fadlullah ZM (2020) Ai aided noise processing of spintronic based iotxf sensor for magnetocardiography 
application. In: ICC 2020-2020 IEEE International Conference on Communications (ICC), pp. 1-6. IEEE

[4] Wang X, Chen Y, Gu Y, Li H (2009) Spintronic memristor temperature sensor. IEEE Electron Device Lett 31(1):20-22

[5] Barla P, Joshi VK, Bhat S (2021) Spintronic devices: a promising alternative to cmos devices. J Comput Electron 20(2):805-837

[6] Makarov A, Windbacher T, Sverdlov V, Selberherr S (2016) Cmos-compatible spintronic devices: a review. Semiconductor Sci Technol 31(11):113006

[7] Sengupta A, Banerjee A, Roy K (2016) Hybrid spintroniccmos spiking neural network with on-chip learning: Devices, circuits, and systems. Phys Rev Appl 6(6):064003

[8] Zhao W, Deng E, Klein JO, Cheng Y, Ravelosona D, Zhang $\mathrm{Y}$, Chappert $\mathrm{C}$ et al (2014) A radiation hardened hybrid spintronic/cmos nonvolatile unit using magnetic tunnel junctions. J Phys D: Appl Phys 47(40):405003

[9] Chen C, Zhang H, Fan Y, Zhang W, Wei R, Wang T, Zhang $\mathrm{T}$, Li F (2020) A novel ultrafine-grained high entropy alloy with excellent combination of mechanical and soft magnetic properties. J Magn Magn Mater 502:166513

[10] Dong Z, Schönecker S, Li W, Chen D, Vitos L (2018) Thermal spin fluctuations in $\mathrm{CoCrFeMnNi}$ high entropy alloy. Sci Rep 8(1):1-7

[11] Koželj P, Vrtnik S, Krnel M, Jelen A, Gačnik D, Wencka M, Jagličić Z, Meden A, Dražić G, Danoix F et al (2021) Spinglass magnetism of the non-equiatomic cocrfemnni highentropy alloy. J Magn Magn Mater 523:167579

[12] Niu C, Zaddach A, Oni A, Sang X, Hurt J III, LeBeau J, Koch C, Irving D (2015) Spin-driven ordering of $\mathrm{cr}$ in the equiatomic high entropy alloy nifecrco. Appl Phys Lett 106(16):161906

[13] Evans RF, Ostler TA, Chantrell RW, Radu I, Rasing T (2014) Ultrafast thermally induced magnetic switching in synthetic ferrimagnets. Appl Phys Lett 104(8):082410

[14] Olejník K, Seifert T, Kašpar Z, Novák V, Wadley P, Campion RP, Baumgartner M, Gambardella P, Němec P, Wunderlich J et al (2018) Terahertz electrical writing speed in an antiferromagnetic memory. Sci Adv 4(3):eaar3566

[15] Ostler T, Barker J, Evans R, Chantrell R, Atxitia U, Chubykalo-Fesenko O, El Moussaoui S, Le Guyader L, Mengotti E, Heyderman L et al (2012) Ultrafast heating as a sufficient stimulus for magnetization reversal in a ferrimagnet. Nat Commun 3(1): $1-6$

[16] Nakayama $\mathrm{H}, \mathrm{Xu} \mathrm{B}$, Iwamoto S, Yamamoto K, Iguchi R, Miura A, Hirai T, Miura Y, Sakuraba Y, Shiomi J et al (2021) Above-room-temperature giant thermal conductivity switching in spintronic multilayers. Appl Phys Lett 118(4):042409
[17] Terakado N, Nara Y, Machida Y, Takahashi Y, Fujiwara T (2020) Dynamic control of heat flow using a spin-chain ladder cuprate film and an ionic liquid. Sci Rep 10(1):1-8

[18] Bauer GE, Saitoh E, Van Wees BJ (2012) Spin caloritronics. Nat Mater 11(5):391-399

[19] Hohenberg P, Kohn W (1964) Inhomogeneous electron gas. Phys. Rev. 136:B864-B871 https://doi.org/10.1103/PhysRe v.136.B864. https://link.aps.org/doi/10.1103/PhysRev.136. B864

[20] Kohn W, Sham LJ (1965) Self-consistent equations including exchange and correlation effects. Phys. Rev. 140:A1133A1138 https://doi.org/10.1103/PhysRev.140.A1133. https://1 ink.aps.org/doi/10.1103/PhysRev.140.A1133

[21] Frey NC, Kumar H, Anasori B, Gogotsi Y, Shenoy VB (2018) Tuning noncollinear spin structure and anisotropy in ferromagnetic nitride MXenes. ACS Nano 12(6):6319-6325

[22] Hobbs D, Hafner J (2001) Ab initio density functional study of phase stability and noncollinear magnetism in mn. J Phys: Condens Matter 13(28):L681

[23] Behera AK, Chowdhury S, Das SR (2019) Magnetic skyrmions in atomic thin CrI3 monolayer. Appl Phys Lett 114(23):232402

[24] Drain JF, Drautz R, Pettifor D (2014) Magnetic analytic bond-order potential for modeling the different phases of Mn at zero kelvin. Phys Rev B 89(13):134102

[25] Evans RF, Fan WJ, Chureemart P, Ostler TA, Ellis MO, Chantrell RW (2014) Atomistic spin model simulations of magnetic nanomaterials. J Phys: Condens Matter 26(10):103202

[26] Kruzik M, Prohl A (2006) Recent developments in the modeling, analysis, and numerics of ferromagnetism. SIAM Rev 48(3):439-483

[27] Ma PW, Woo C, Dudarev S (2008) Large-scale simulation of the spin-lattice dynamics in ferromagnetic iron. Phys Rev B 78(2):024434

[28] Woo C, Wen H, Semenov A, Dudarev S, Ma PW (2015) Quantum heat bath for spin-lattice dynamics. Phys Rev B 91(10): 104306

[29] Zhou Y, Tranchida J, Ge Y, Murthy J, Fisher TS (2020) Atomistic simulation of phonon and magnon thermal transport across the ferromagnetic-paramagnetic transition. Phys Rev B 101(22):224303

[30] Ge Y, Zhou Y, Fisher TS (2021) Thermal boundary conductance across $\mathrm{Co} / \mathrm{Cu}$ interfaces with spin-lattice interactions. J Appl Phys 130(23):235108

[31] Wu X, Liu Z, Luo T (2018) Magnon and phonon dispersion lifetime and thermal conductivity of iron from spin-lattice dynamics simulations. J Appl Phys 123(8):085109. https://d oi.org/10.1063/1.5020611 
[32] Kubo R (1966) The fluctuation-dissipation theorem. Rep Prog Phys 29(1):255

[33] Tranchida J, Plimpton S, Thibaudeau P, Thompson AP (2018) Massively parallel symplectic algorithm for coupled magnetic spin dynamics and molecular dynamics. J Comput Phys 372:406-425

[34] Beaujouan D, Thibaudeau P, Barreteau C (2012) Anisotropic magnetic molecular dynamics of cobalt nanowires. Phys Rev B 86(17):174409-174420. https://doi.org/10.1103/PhysRev B.86.174409

[35] Nieves P, Tranchida J, Arapan S, Legut D (2021) Spinlattice model for cubic crystals. Physical Review B 103(9):094437. https://doi.org/10.1103/PhysRevB.103. 094437

[36] Zuo Y, Chen C, Li X, Deng Z, Chen Y, Behler J, Csányi G, Shapeev AV, Thompson AP, Wood MA et al (2020) Performance and cost assessment of machine learning interatomic potentials. J Phys Chem A 124(4):731-745

[37] Nikolov S, Wood MA, Cangi A, Maillet JB, Marinica MC, Thompson AP, Desjarlais MP, Tranchida J (2021) Data-driven magneto-elastic predictions with scalable classical spinlattice dynamics. Npj Comput Mater 7:153. https://doi.org/ 10.1038/s41524-021-00617-2

[38] Plimpton S (1995) Fast parallel algorithms for short-range molecular dynamics. J Comput Phys 117(1):1-19

[39] Kresse G, Furthmüller J (1996) Efficient iterative schemes for ab initio total-energy calculations using a plane-wave basis set. Phys. Rev. B 54:11169-11186 https://doi.org/10. 1103/PhysRevB.54.11169. https://link.aps.org/doi/10.1103/ PhysRevB.54.11169

[40] Kresse G, Hafner J (1993) Ab initio molecular dynamics for liquid metals. Phys. Rev. B 47:558-561 https://doi.org/10. 1103/PhysRevB.47.558. https://link.aps.org/doi/10.1103/Ph ysRevB.47.558

[41] Kresse G, Joubert D (1999) From ultrasoft pseudopotentials to the projector augmented-wave method. Phys. Rev. B 59:1758-1775 https://doi.org/10.1103/PhysRevB.59.1758. h ttps://link.aps.org/doi/10.1103/PhysRevB.59.1758

[42] Kresse G, Furthmüller J (1996) Efficiency of ab-initio total energy calculations for metals and semiconductors using a plane-wave basis set. Comput Mater Sci 6(1):15-50. http s://doi.org/10.1016/0927-0256(96)00008-0

[43] Lin KH, Strachan A (2013) Thermal transport in SiGe superlattice thin films and nanowires: Effects of specimen and periodic lengths. Phys Rev B 87(11):115302

[44] Müller-Plathe F (1997) A simple nonequilibrium molecular dynamics method for calculating the thermal conductivity. J Chem Phys 106(14):6082-6085

[45] Lussetti E, Terao T, Müller-Plathe F (2007) Nonequilibrium molecular dynamics calculation of the thermal conductivity of amorphous polyamide-6, 6. J Phys Chem B 111(39):11516-11523

[46] Terao T, Lussetti E, Müller-Plathe F (2007) Nonequilibrium molecular dynamics methods for computing the thermal conductivity: Application to amorphous polymers. Phys Rev E 75(5):057701

[47] Evans RF, Atxitia U, Chantrell RW (2015) Quantitative simulation of temperature-dependent magnetization dynamics and equilibrium properties of elemental ferromagnets. Phys Rev B 91(14):144425

[48] Greenwood D (1958) The Boltzmann equation in the theory of electrical conduction in metals. Proc Phys Soc 71(4):585

[49] Kubo R (1957) Statistical-mechanical theory of irreversible processes. I. General theory and simple applications to magnetic and conduction problems. J Phys Soc Japan 12(6):570-586

[50] Mahan GD (2013) Many-particle physics. Springer, Berlin

[51] Desjarlais MP, Kress JD, Collins LA (2002) Electrical conductivity for warm dense aluminum plasmas and liquids. Phys Rev E 66(2):025401.

[52] Blöchl PE (1994) Projector augmented-wave method. Phys Rev B 50(24):17953

[53] Perdew JP, Burke K, Ernzerhof M (1996) Generalized gradient approximation made simple. Phys Rev Lett 77(18):3865

[54] Kubo-greenwood electronic transport calculations with vasp code. https://github.com/conodipaola/kg4vasp

[55] Di Paola C, Macheda F, Laricchia S, Weber C, Bonini N (2020) First-principles study of electronic transport and structural properties of $\mathrm{cu}_{12} \mathrm{sb}_{4} \mathrm{~s}_{13}$ in its high-temperature phase. Phys. Rev. Research 2:033055 https://doi.org/10.110 3/PhysRevResearch.2.033055. https://link.aps.org/doi/10.11 03/PhysRevResearch.2.033055

[56] Fulkerson W, Moore J, McElroy D (1966) Comparison of the Thermal Conductivity, Electrical Resistivity, and Seebeck Coefficient of a High-Purity Iron and an Armco Iron to 1000 C. J Appl Phys 37(7):2639-2653

[57] Bäcklund N (1961) An experimental investigation of the electrical and thermal conductivity of iron and some dilute iron alloys at temperatures above $100 \mathrm{k}$. J Phys Chem Solids 20(1-2):1-16

[58] Korell JA, French M, Steinle-Neumann G, Redmer R (2019) Paramagnetic-to-diamagnetic transition in dense liquid iron and its influence on electronic transport properties. Phys Rev Lett 122(8):086601

[59] Pozzo M, Davies C, Gubbins D, Alfe D (2012) Thermal and electrical conductivity of iron at earth's core conditions. Nature 485(7398):355-358 
[60] Pozzo M, Davies C, Gubbins D, Alfè D (2014) Thermal and electrical conductivity of solid iron and iron-silicon mixtures at earth's core conditions. Earth Planet Sci Lett 393:159-164

[61] Mao H, Xu J, Struzhkin V, Shu J, Hemley R, Sturhahn W, Hu M, Alp E, Vocadlo L, Alfè D et al (2001) Phonon density of states of iron up to 153 gigapascals. Science 292(5518):914-916

[62] Minkiewicz VJ, Shirane G, Nathans R (1967) Phonon dispersion relation for iron. Phys. Rev. 162:528-531 https://doi. org/10.1103/PhysRev.162.528. https://link.aps.org/doi/10.11 03/PhysRev.162.528

[63] Hu M, Sturhahn W, Toellner T, Hession P, Sutter J, Alp E (1999) Data analysis for inelastic nuclear resonant absorption experiments. Nuclear Instruments and Methods in Physics Research Section A: Accelerators, Spectrometers, Detectors and Associated Equipment 428(2):551-555 http s://doi.org/10.1016/S0168-9002(99)00134-5. https://www.sc iencedirect.com/science/article/pii/S0168900299001345

[64] Kresch M, Delaire O, Stevens R, Lin J, Fultz B (2007) Neutron scattering measurements of phonons in nickel at elevated temperatures. Phys Rev B 75(10):104301

[65] Mauger L, Lucas M, Muñoz J, Tracy S, Kresch M, Xiao Y, Chow P, Fultz B (2014) Nonharmonic phonons in $\alpha$-iron at high temperatures. Phys Rev B 90(6):064303

[66] Körmann F, Grabowski B, Dutta B, Hickel T, Mauger L, Fultz B, Neugebauer J (2014) Temperature dependent magnon-phonon coupling in bcc Fe from theory and experiment. Phys Rev Lett 113(16):165503. https://doi.org/10.1103/Phy sRevLett.113.165503

[67] Strungaru M, Ellis MO, Ruta S, Chubykalo-Fesenko O, Evans RF, Chantrell RW (2021) Spin-lattice dynamics model with angular momentum transfer for canonical and microcanonical ensembles. Physical Review B 103:024429. http s://doi.org/10.1103/PhysRevB.103.024429

[68] Skomski R (2008) Simple models of magnetism. Oxford University Press, New York

[69] Rosengaard N, Johansson B (1997) Finite-temperature study of itinerant ferromagnetism in Fe, Co, and Ni. Phys Rev B 55(22): 14975

[70] Szilva A, Costa M, Bergman A, Szunyogh L, Nordström L, Eriksson O (2013) Interatomic exchange interactions for finite-temperature magnetism and nonequilibrium spin dynamics. Phys Rev Lett 111(12):127204

[71] Kaneyoshi T (1992) Introduction to amorphous magnets. World Scientific Publishing Company, Singapore

[72] Yosida K, Mattis DC, Yosida K (1996) Theory of Magnetism.: Edition en anglais, vol. 122. Springer Science \& Business Media

[73] Yang KH, Hirschfelder JO (1980) Generalizations of classical poisson brackets to include spin. Phys Rev A 22(5): 1814

[74] Evans RF (2020) Atomistic spin dynamics. Handbook of Materials Modeling: Applications: Current and Emerging Materials pp. $427-448$

[75] Zhang IY, Xu X, Jung Y, Goddard WA (2011) A fast doubly hybrid density functional method close to chemical accuracy using a local opposite spin ansatz. Proc Nat Academy Sci 108(50):19896-19900

Publisher's Note Springer Nature remains neutral with regard to jurisdictional claims in published maps and institutional affiliations. 